\title{
Charge transfer-induced magnetic exchange bias and electron localization in (111)- and (001)-oriented $\mathrm{LaNiO}_{3} / \mathrm{LaMnO}_{3}$ superlattices
}

Haoming Wei, Jose Luis Barzola-Quiquia, Chang Yang, Christian Patzig, Thomas Höche, Pablo Esquinazi, Marius Grundmann, and Michael Lorenz

Citation: Appl. Phys. Lett. 110, 102403 (2017); doi: 10.1063/1.4978358

View online: https://doi.org/10.1063/1.4978358

View Table of Contents: http://aip.scitation.org/toc/apl/110/10

Published by the American Institute of Physics

\section{Articles you may be interested in}

Confinement-driven metal-insulator transition and polarity-controlled conductivity of epitaxial $\mathrm{LaNiO}_{3} / \mathrm{LaAlO}_{3}$ (111) superlattices

Applied Physics Letters 109, 082108 (2016); 10.1063/1.4961693

Electronic structure of buried $\mathrm{LaNiO}_{3}$ layers in (111)-oriented $\mathrm{LaNiO}_{3} / \mathrm{LaMnO}_{3}$ superlattices probed by soft $x$-ray ARPES

APL Materials 5, 016101 (2017); 10.1063/1.4973558

Comparative study of $\mathrm{LaNiO}_{3} / \mathrm{LaAlO}_{3}$ heterostructures grown by pulsed laser deposition and oxide molecular beam epitaxy

Applied Physics Letters 110, 041606 (2017); 10.1063/1.4975005

Antisite disorder induced spin glass and exchange bias effect in $\mathrm{Nd}_{2} \mathrm{NiMnO}_{6}$ epitaxial thin film Applied Physics Letters 110, 102402 (2017); 10.1063/1.4978354

Ultra-low magnetic damping of perovskite $\mathrm{La}_{0.7} \mathrm{Sr}_{0.3} \mathrm{MnO}_{3}$ thin films Applied Physics Letters 110, 112401 (2017); 10.1063/1.4978431

Room temperature insulating ferromagnetism induced by charge transfer in ultrathin (110) $\mathrm{La}_{0.7} \mathrm{Sr}_{0.3} \mathrm{MnO}_{3}$ films

Applied Physics Letters 110, 072405 (2017); 10.1063/1.4976699

\section{Conference Proceedings}




\title{
Charge transfer-induced magnetic exchange bias and electron localization in (111)- and (001)-oriented $\mathrm{LaNiO}_{3} / \mathrm{LaMnO}_{3}$ superlattices
}

\author{
Haoming Wei, ${ }^{1}$ Jose Luis Barzola-Quiquia, ${ }^{1}$ Chang Yang, ${ }^{1}$ Christian Patzig, ${ }^{2}$ \\ Thomas Höche, ${ }^{2}$ Pablo Esquinazi, ${ }^{1}$ Marius Grundmann, ${ }^{1}$ and Michael Lorenz ${ }^{1, a)}$ \\ ${ }^{1}$ Felix-Bloch-Institut für Festkörperphysik, Universität Leipzig, Linnéstr. 5, D-04103 Leipzig, Germany \\ ${ }^{2}$ Center für angewandte Mikrostrukturdiagnostik, Fraunhofer-Institut für Mikrostruktur von Werkstoffen \\ und Systemen, Walter-Hülse-Straße 1, D-06120 Halle, Germany
}

(Received 13 January 2017; accepted 25 February 2017; published online 9 March 2017)

\begin{abstract}
High-quality lattice-matched $\mathrm{LaNiO}_{3} / \mathrm{LaMnO}_{3}$ superlattices with monolayer terrace structure have been grown on both (111)- and (001)-oriented $\mathrm{SrTiO}_{3}$ substrates by pulsed laser deposition. In contrast to the previously reported experiments, a magnetic exchange bias is observed that reproducibly occurs in both (111)- and (001)-oriented superlattices with the thin single layers of 5 and 7 unit cells, respectively. The exchange bias is theoretically explained by charge transfer-induced magnetic moments at $\mathrm{Ni}$ atoms. Furthermore, magnetization data at low temperature suggest two magnetic phases in the superlattices, with Néel temperature around $10 \mathrm{~K}$. Electrical transport measurements reveal a metal-insulator transition with strong localization of electrons in the superlattices with the thin $\mathrm{LaNiO}_{3}$ layers of 4 unit cells, in which the electrical transport is dominated by two-dimensional variable range hopping. Published by AIP Publishing. [http://dx.doi.org/10.1063/1.4978358]
\end{abstract}

Transition metal oxides provide a fertile ground for the realization of device functionalities due to multiple degrees of freedom in their charge, spin and orbital states. In particular, in superlattices (SLs), modification of the band structure through the design of artificial heterostructures gives rise to a variety of phenomena such as exchange bias (EB), Mott insulation, superconductivity, and topological and Chern insulation. ${ }^{1-3}$ The EB effect, exhibiting a shift of the center of the magnetic hysteresis loop along the magnetic field axis, is one of the outcomes of exchange anisotropy at the interface between two materials with competing magnetic interactions. ${ }^{4}$ This effect is well known in many different systems containing interfaces between ferromagnetic and antiferromagnetic materials. ${ }^{5}$

In 2012, Gibert et al. reported an unexpected EB effect in SLs composed of (111)-oriented layers of paramagnetic $\mathrm{LaNiO}_{3}$ (LNO) and ferromagnetic $\mathrm{LaMnO}_{3}$ (LMO) on (111) $\mathrm{SrTiO}_{3}{ }^{6}{ }^{6}$ However, the authors declare the absence of $\mathrm{EB}$ in (001)-oriented LNO/LMO SLs (grown on (001) $\left.\mathrm{SrTiO}_{3}\right)^{6}$ This interesting phenomenon has stimulated further theoretical work to explain the EB effects for different orientations of the SLs. ${ }^{7-9}$ Dong and Dagotto claim "the induced magnetization is largest for the (111)-stacking and the weakest for the (001)-stacking superlattices.." Lee and Han investigated the electronic structure and magnetic properties of LNO/ LMO SLs using the first-principles calculations in the framework of density functional theory. ${ }^{9}$ They concluded that the magnetic moments at $\mathrm{Ni}$ sites are induced by charge transfer between $\mathrm{Ni}$ and $\mathrm{Mn}$ at the interface, with only minor differences for (111)- and (001)-oriented SLs. ${ }^{9}$ In addition, it was found that the couplings between $\mathrm{Ni}-\mathrm{Ni}$ and $\mathrm{Mn}-\mathrm{Mn}$ atoms, in some cases, could introduce an antiferromagnetic phase in the SLs. ${ }^{9}$ Based on these theoretical studies, the EB effect should be mostly independent of the crystallographic orientation of the LNO/LMO SLs and possibly exists in other

\footnotetext{
${ }^{\text {a) }}$ Author to whom correspondence should be addressed. Electronic mail: mlorenz@physik.uni-leipzig.de
}

kinds of artificial heterostructures with charge transferinduced magnetic moments.

However, up to now, a broader experimental database is missing because the growth of high-quality LNO/LMO (111) SLs is still a challenge due to the highly polar atomic layers along the [111] direction. ${ }^{10}$ In addition, studies of electronic transport properties of LNO/LMO SLs are lacking due to the possible occurrence of complex surface reconstruction caused by the polarity compensation. ${ }^{11} \mathrm{~A}$ better understanding of the underlying physics could promote applications of the LNO/ LMO SLs in magnetic recording, magnetic tunnel junctions, and giant magnetoresistance sensors. ${ }^{12}$ In this paper, we report on the epitaxial growth of $\left[\mathrm{LNO}_{m} / \mathrm{LMO}_{n}\right]_{l}$ SLs ( $m$ and $n$ indicate the number of unit cells, respectively, thereafter referred to as $\left.[\mathrm{m} / \mathrm{n}]_{l}\right)$ in both (111) and (001) orientations. The EB effect is observed reproducibly in several SLs for both orientations, with different stacking periodicity $l$ from 7 to 13 , and for single layer thicknesses $m, n$ of 5 or 7 unit cells. Furthermore, metal-insulator transition and charge transfer-induced electron localization have been observed in other LNO/LMO SLs.

High-quality lattice matched SLs were grown by pulsed laser deposition (PLD) using a $\mathrm{KrF}$ excimer laser with growth control by in-situ reflection high-energy electron diffraction (RHEED). Stoichiometric LNO and LMO targets were prepared by mixing high-purity $\mathrm{La}_{2} \mathrm{O}_{3}$ and $\mathrm{NiO}$ or $\mathrm{MnO}$ powders, pressing into a 1 -inch diameter pellet and sintering. $\mathrm{SrTiO}_{3}$ (STO) single crystals with (001) and (111) orientation and low miscut below $0.15^{\circ}$ were selected as substrates. Before the PLD process, substrates were etched in diluted hydrogen fluoride solution and annealed under oxygen atmosphere in order to obtain a surface terminated with terraced monolayer steps. PLD growth was done at temperatures of about $680^{\circ} \mathrm{C}$ and oxygen pressures of 0.05 mbar for LNO and $3 \times 10^{-4}$ mbar for LMO. The single layer thicknesses were adjusted by the number of applied laser pulses. After deposition, samples were annealed in-situ in $800 \mathrm{mbar}$ oxygen at growth temperature for $15 \mathrm{~min}$. 
The out-of-plane orientation and in-plane epitaxial relationship between the SLs and their substrates were examined by X-ray diffraction (XRD) reciprocal space maps (RSMs). RSMs were recorded with a PANalytical X'pert PRO Materials Research Diffractometer using $\mathrm{Cu}-\mathrm{K} \alpha$ radiation from a parabolic mirror and a PIXcel ${ }^{3 \mathrm{D}}$ multichannel detector. The surface morphology of SLs was investigated by atomic force microscopy (AFM) in dynamic non-contact mode (Park Systems XE-150). The nanostructures of the SLs were imaged in cross-section using a $\mathrm{c}_{\mathrm{s}}$-aberration corrected high-resolution transmission electron microscope (HR-TEM) FEI TITAN $\mathrm{G}^{2} 80-300$, operated at $300 \mathrm{kV}$, equipped with a SuperX-EDX detection system (FEI company) for the highly efficient recording of energy-dispersive X-Ray spectroscopy (EDS). The magnetic properties of SLs were measured using a superconducting quantum interference device (SQUID) magnetometer MPMS-7 and double-checked by the vibrating sample magnetometer (VSM) of a physical property measurement system (PPMS-9), both from Quantum Design, Inc. The in-plane resistivity was measured as a function of temperature in four-point van der Pauw geometry with DCsputtered ohmic gold contacts at the corners of the samples, using Keithley Hall effect electronics.

The growth of LNO single films and $\mathrm{LNO} / \mathrm{LaAlO}_{3}$ SLs has already been reported by us (see Refs. 13 and 14). In this work, the growth of LMO films is investigated first. As shown in the inset of Figure 1(a), a sharp RHEED pattern was observed during LMO growth. The RHEED patterns, taken along $\langle 110\rangle$ STO direction, show a high intensity specular spot with Kikuchi lines. The evolution of the intensity of the specular spot was monitored during LMO growth, as shown in Fig. 1(a). The oscillations almost completely maintain their amplitude during the growth of 10 monolayers of LMO, signalizing a two-dimensional (2D) layer-by-layer growth. The sharp RHEED pattern after deposition $(t=220 \mathrm{~s})$ confirms an atomically flat film surface. Fig. 1(b) displays a typical AFM image of a $[2 / 2]_{6}$ SL deposited on STO (001) substrate. The SL exhibits terraces with monolayer steps and low roughness of $0.095 \mathrm{~nm}$. The step height is around $0.39 \mathrm{~nm}$, corresponding to one unit cell in the (001) orientation. A similar monolayer terraced surface with an average step height of $0.22 \mathrm{~nm}$ is also observed for the (111)-oriented SLs, as shown in Fig. S1 in supplementary material online.

The out-of-plane crystallographic orientations of SLs are determined as (001) and (111) from the reflections around symmetric peaks of (002) and (111), as shown in Fig. 1(c) and Fig. S2(a) (supplementary material), respectively. The inplane lattice match (pseudomorphic growth) can be deduced from the vertical alignment of the asymmetric SL and substrate peaks, as shown in Fig. 1(d) for (001) SL and Fig. S2(b) (supplementary material) for (111) SL. In addition, the SL period has been calculated from the distance between adjacent satellite peaks using the relationship $L=\lambda /\left(2 \Delta \theta \cdot \cos \theta_{B}\right)$, where $\lambda$ is the $\mathrm{X}$-ray wavelength, $\Delta \theta$ is the angular separation between two adjacent satellite peaks, and $\theta_{B}$ is the Bragg angle of the zero-order satellite peak. ${ }^{15}$

More detailed structural insights are obtained by HRTEM. Fig. 2(a) shows an overview TEM cross section image of the $[7 / 7]_{10} \mathrm{LNO} / \mathrm{LMO}$ SLs grown on STO (111) substrates. The interfaces between the LNO and LMO layers are clearly visible and coherent over a wide lateral range. An integrated EDS line scan was taken along the growth direction. The profiles of the intensities of the $\mathrm{Mn}-\mathrm{K}_{\alpha}$ (black) and $\mathrm{Ni}-\mathrm{K}_{\alpha}$ (red) are plotted in Fig. 2(c). Ten double layers LNO/LMO with the thickness of about $3 \mathrm{~nm}$ were confirmed, corresponding to the designed $[7 / 7]_{10}$ SL structure. An HR-TEM image shown in Fig. 2(b) further confirms the epitaxial in-plane lattice match and coherent interfaces of the SL. The inter-planar spacing along the growth direction is $0.22 \mathrm{~nm}$, a value equal to the step height of a single unit cell measured by AFM. The in-

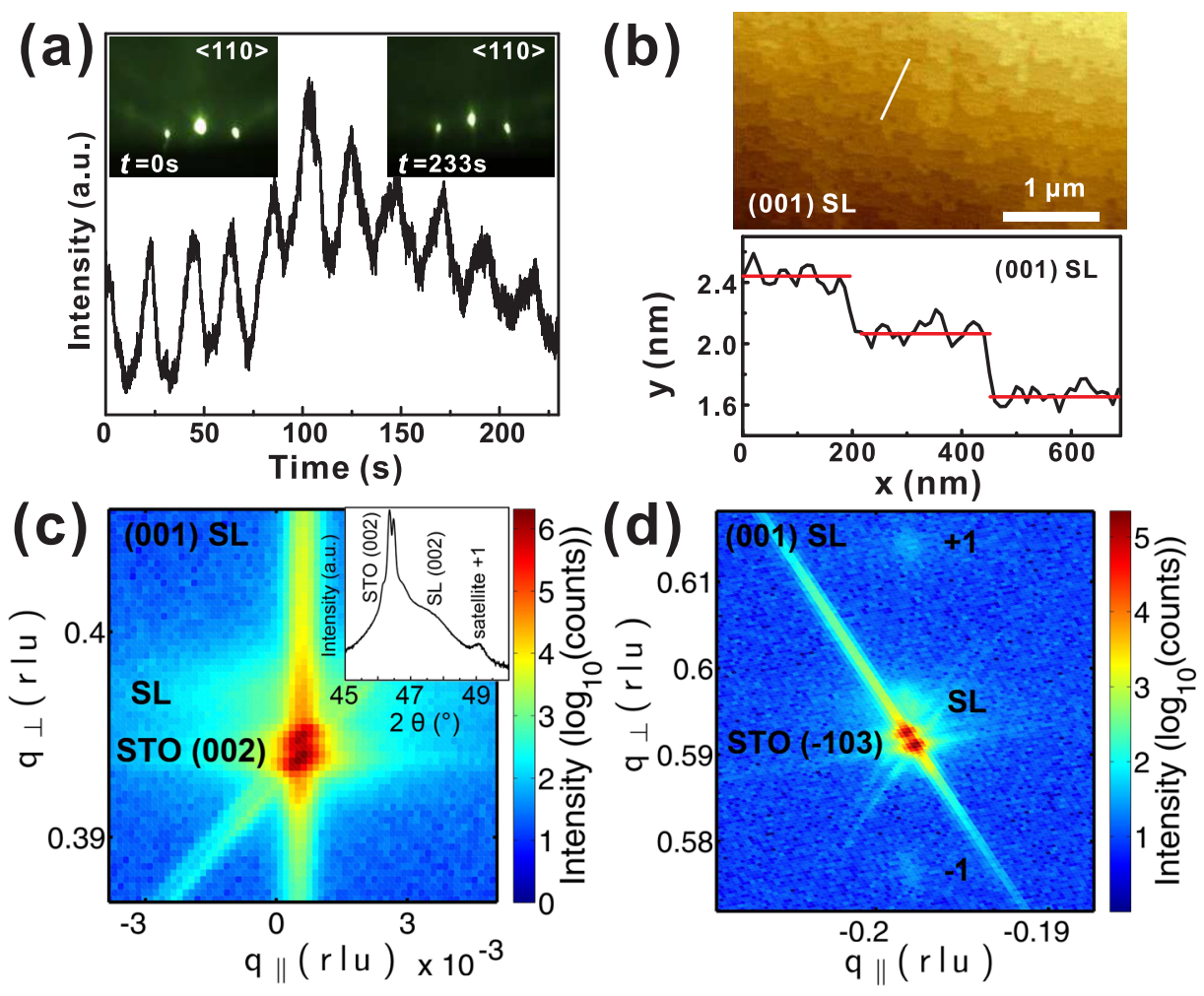

FIG. 1. (a) RHEED oscillations of 10 monolayers LMO grown on STO (001) substrate. The insets are in-situ RHEED patterns before and after deposition. (b) AFM image of LNO/LMO $[2 / 2]_{6}$ SL on STO (001) substrate. Steps with a height of one unit cell are observed in the scan along the short white line. The red lines are guides to the eyes. (c) and (d) XRD RSMs of [8/2] $]_{10}$ (001) SLs around the symmetric (002) (c) and asymmetric $(-103)$ (d) reflexes. The double-peak structure of substrates is caused by $\mathrm{K} \alpha_{1 / 2}$ splitting of the incident $\mathrm{X}$-ray beam. rlu stands for reciprocal lattice unit. 

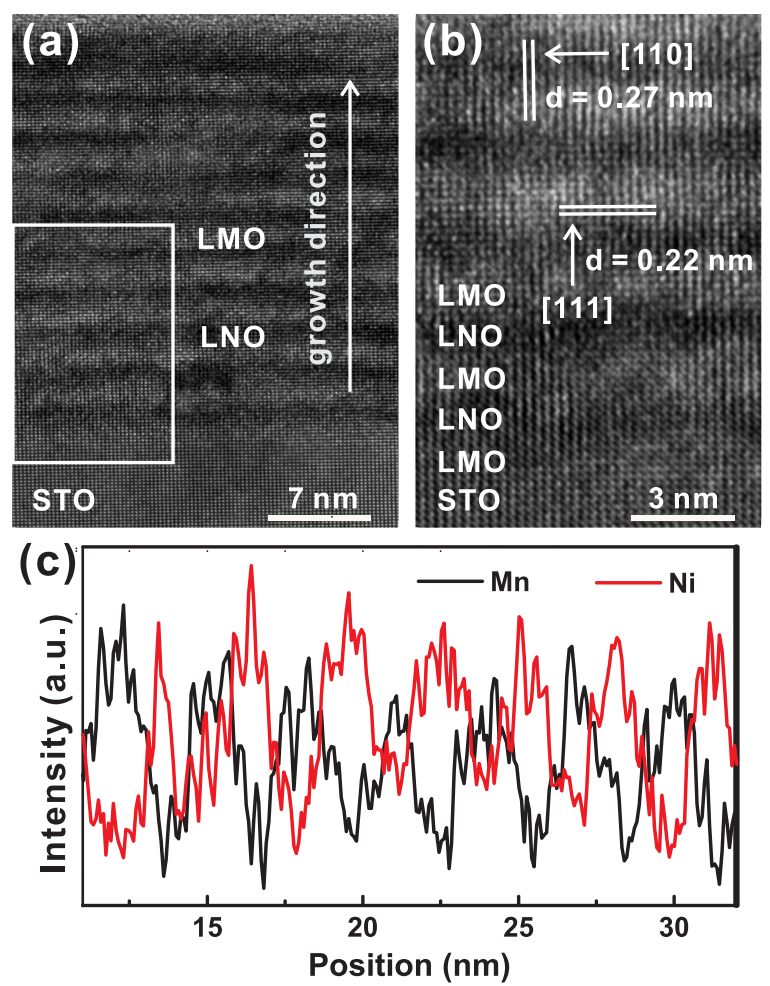

FIG. 2. HR-TEM images of the $[7 / 7]_{10}$ SL grown on STO (111) substrate: (a) overview and (b) white square from (a) at higher magnification. The LMO layers appear brighter than the LNO layers. (c) Integrated EDS line scan along the growth direction. The profiles of the intensities of $\mathrm{Mn}-\mathrm{K}_{\alpha}$ (black) and $\mathrm{Ni}-\mathrm{K}_{\alpha}$ (red) indicate the chemical superlattice structure.

plane lattice parameter of SL is about $0.27 \mathrm{~nm}$, further confirming the lattice match between SL and substrate.

The magnetic properties were measured with the field applied in-plane to the SLs. The total thickness of both demonstrated SLs is around $41 \mathrm{~nm}$, which was adjusted via the number of superlattice periods. Note that one unit cell corresponds to lower values in (111) orientation in comparison to (001). Fig. 3(a) presents hysteresis loops of (111)-oriented
SL at $2 \mathrm{~K}$ after field-cooling (FC) from room temperature in the presence of $\pm 1 \mathrm{~T}$ fields. The shift of the hysteresis loops along the magnetic field axis is clearly observed. This behavior is the typical signature of the EB effect. When the temperature increases to $10 \mathrm{~K}$, the $\mathrm{EB}$ effect is still evident in Fig. S3(a) (supplementary material). For (001)-oriented SLs, we have also observed the EB effect, as shown in Fig. 3(b) and quantified in Table I. This observation indicates that the EB seems to be an intrinsic property of LNO/LMO interfaces in the SLs and is relatively independent of crystallographic orientation. This experimental finding is in agreement with the theoretical predictions from Ref. 6.

However, evaluating the temperature dependence of EB field $H_{E}$ in Table I, we noted that the values for (001) systematically decreased strongly with temperature as for (111) orientation. The EB effect gradually vanishes for temperatures above $6 \mathrm{~K}$ for (001) SL and above $10 \mathrm{~K}$ for (111) SL, corresponding to the Néel temperatures shown in Figs. 3(c) and S3(c) (supplementary material), respectively. The EB field of (111)-oriented SL finally vanishes in between 40 and $50 \mathrm{~K}$, i.e., close to the blocking temperature that will be discussed further below.

The EB effect can be quantitatively analyzed in terms of the EB field, $H_{E}$, which is given by $H_{E}=\left(H_{C}^{+}+H_{C}^{-}\right) / 2$, where $H_{C}^{+}$and $H_{C}^{-}$denote the positive and negative coercive fields at which the magnetization equals zero. ${ }^{16}$ The $H_{E}$ values of (111)- and (001)-oriented SLs are summarized in Table I. It is temperature dependent and clearly decreases with increasing temperature. The $H_{E}$ is typically accompanied by an enhancement of the average coercive field $H_{C}$, which is given by $H_{C}=\left(\left|H_{C}^{+}\right|+\left|H_{C}^{-}\right|\right) / 2 .{ }^{17}$ We observed a monotonic decrease in coercive field with an increase in temperature. This can be understood from considering the effects of thermal fluctuations of the blocked moment across the anisotropy barrier. ${ }^{18,19}$ Thus, the $H_{C}$ is linear to $\mathrm{T}^{1 / 2}$ $\left(\right.$ Kneller's law ${ }^{20}$ ), as shown in Fig. S3(b) (supplementary material) for both (001)- and (111)-oriented SLs.

It is worth to mention that there is a possibility that (111)oriented facets can occur in (001) SLs due to locally rough


FIG. 3. (a) Magnetic hysteresis loops at $2 \mathrm{~K}$ for the (111)-oriented [7/7] $]_{13} \mathrm{SL}$ after field-cooling at two different fields $\mu_{0} \mathrm{H}_{\mathrm{FC}}= \pm 1 \mathrm{~T}$. (b) $\mathrm{Hysteresis}$ loops for the (001)-oriented $[5 / 5]_{10}$ SL at different constant temperatures after cooling the sample with a field of $+1 \mathrm{~T}$. (c) Magnetic moment versus temperature of (001)-oriented SL in the ZFC and FC states at a field of $0.2 \mathrm{~T}$. The inset is dM/dT versus temperature of FC curve, and its minimum is close to the Curie temperature. Loops (a) and (b) are measured with SQUID and (c) is from VSM of PPMS. 
TABLE I. The EB field $H_{E}$ and coercive field $H_{C}$ of [7/7 $]_{13}$ (111) SL and [5/ $5]_{10}(001)$ SL at different temperatures.

\begin{tabular}{lccccc}
\hline \hline & \multicolumn{2}{c}{$(111)$-oriented SL } & & \multicolumn{2}{c}{$(001)$-oriented SL } \\
\cline { 2 - 3 } \cline { 6 - 6 } Temperature (K) & $H_{E}(\mathrm{mT})$ & $H_{C}(\mathrm{mT})$ & & $H_{E}(\mathrm{mT})$ & $H_{C}(\mathrm{mT})$ \\
\hline 2 & 23.7 & 143.6 & & 23.1 & 79.5 \\
4 & 4.8 & 104.7 & & 4.2 & 59.7 \\
6 & 3.4 & 75.5 & & 0.3 & 45.7 \\
8 & 2.9 & 55.0 & & 0.2 & 34.5 \\
10 & 2.5 & 41.5 & & 0.2 & 27.8 \\
20 & 1.3 & 17.1 & & $\ldots$ & $\ldots$ \\
30 & 1.2 & 10.9 & & $\ldots$ & $\ldots$ \\
40 & 0.7 & 6.6 & & $\ldots$ & $\ldots$ \\
50 & 0.1 & 3.5 & & $\ldots$ & $\ldots$ \\
\hline \hline
\end{tabular}

interfaces, e.g., at the sides of steps. However, as listed in Table I, both (001) and (111) SLs have large exchange bias fields with only slight differences at $2 \mathrm{~K}$ and $4 \mathrm{~K}$. Thus, the existence of a few (111) facets in (001) SLs is most probably not a dominant factor for the observation of exchange bias effect. In order to further understand the EB effect in the SLs, the zero-field-cooling (ZFC) and FC magnetization measurements have been performed, as shown in Fig. 3(c) for (001)orientation and in Fig. S3(c) (supplementary material) for (111)-orientation. In a field of $0.2 \mathrm{~T}$, the two curves merge at the blocking temperature of about $45 \mathrm{~K}$ that can be seen in Fig. 3(c). At low temperatures, the ZFC and FC curves show different behavior suggesting that the sample contains two magnetic phases. Here, the value of Néel temperature is estimated to be $6 \mathrm{~K} .{ }^{21}$ In addition, the paramagnetic-ferromagnetic phase-transition temperature was found at around $85 \mathrm{~K}$ from the minimum of the $\mathrm{dM} / \mathrm{dT}$ curve, as shown in the insets of Figs. 3(c) and S3(c) (supplementary material). Since the difference in the magnetic moment measured in ZFC and FC sequences was insignificant in SLs built from magnetic LMO and dielectric $\mathrm{LaAlO}_{3}$ (not shown here), we conclude that the EB observed in LNO/LMO SLs is related to the interfaces between the two magnetic components.

The formation of magnetic moments at $\mathrm{Ni}$ sites can be explained by the charge transfer between $\mathrm{Ni}$ and $\mathrm{Mn}$ at the interface. ${ }^{9}$ The $\mathrm{Ni}^{2+}$, formed by receiving one electron from $\mathrm{Mn}$, is expected to have antiferromagnetic coupling to neighboring $\mathrm{Ni}^{2+}$ as in $\mathrm{La}_{2} \mathrm{NiO}_{4}{ }^{22}$ Thus, the co-existence of ferromagnetic coupling between $\mathrm{Ni}-\mathrm{Mn}$ and antiferromagnetic coupling between Ni-Ni leads to the pinning of magnetization and EB effect in the LNO/LMO SLs. ${ }^{9}$ Consequently, our observation suggests that the EB effect is induced by the charge transfer rather than dominated by the crystallographic orientation. A similar EB phenomenon has also been observed in the (001)-oriented $\mathrm{La}_{0.75} \mathrm{Sr}_{0.25} \mathrm{MnO}_{3} / \mathrm{LaNiO}_{3}$ multilayers.

Another two interesting phenomena related to the charge transfer in the LNO/LMO SLs are metal-insulator transition and electron localization, as shown in Fig. 4 and Fig. S4 (supplementary material), respectively. The SLs with the LNO layer of 6 unit cells show metallic behavior with positive sheet resistance temperature coefficient. At high temperatures $(100-300 \mathrm{~K})$, the sheet resistance is linearly proportional to temperature due to dominant electron-phonon scattering, which corresponds well with the reported results on other LNO single films. ${ }^{23}$ With the decreasing number of LNO unit cells in the SLs, insulating behavior appears. The metal-insulator transition has been reported in LNO ultra-thin films, in which the charge disproportionation with an accompanying symmetry change was considered the origin of this phenomenon. However, the precise mechanism for the metal-insulator transition in the LNO-based SLs is still under debate. ${ }^{24}$ There are two scenarios that can be considered for the intrinsic conductivity:

The first scenario takes quantum confinement into account, and the metal-insulator transition depends on the dimension of the LNO layers in the SLs. ${ }^{13,25}$ According to the Boltzmann transport theory, the conductivity of a 2D electron gas is related to the wave number of electrons on the Fermi surface and the mean free path. ${ }^{26}$ Thus, the calculated maximum sheet resistance of a metallic conductor approaches $h /$ $e^{2} \approx 25.8 \mathrm{k} \Omega / \square\left(\underline{\wedge} \mathrm{k} \Omega / \mathrm{m}^{2}\right),{ }^{27}$ which is the quantum of resistance in 2D state, as shown with black dotted lines in Fig. 4(a). Above this value, the SLs exhibit insulating behavior. In the second scenario, the charge redistribution in the LNO/ LMO SLs with an accompanying localization of electrons is considered the origin of the metal-insulator transition. ${ }^{22}$ Similar metal-insulator transition behavior has also been observed in the LNO thin films by changing the valance state of $\mathrm{Ni}$ from trivalent to divalent. ${ }^{28}$
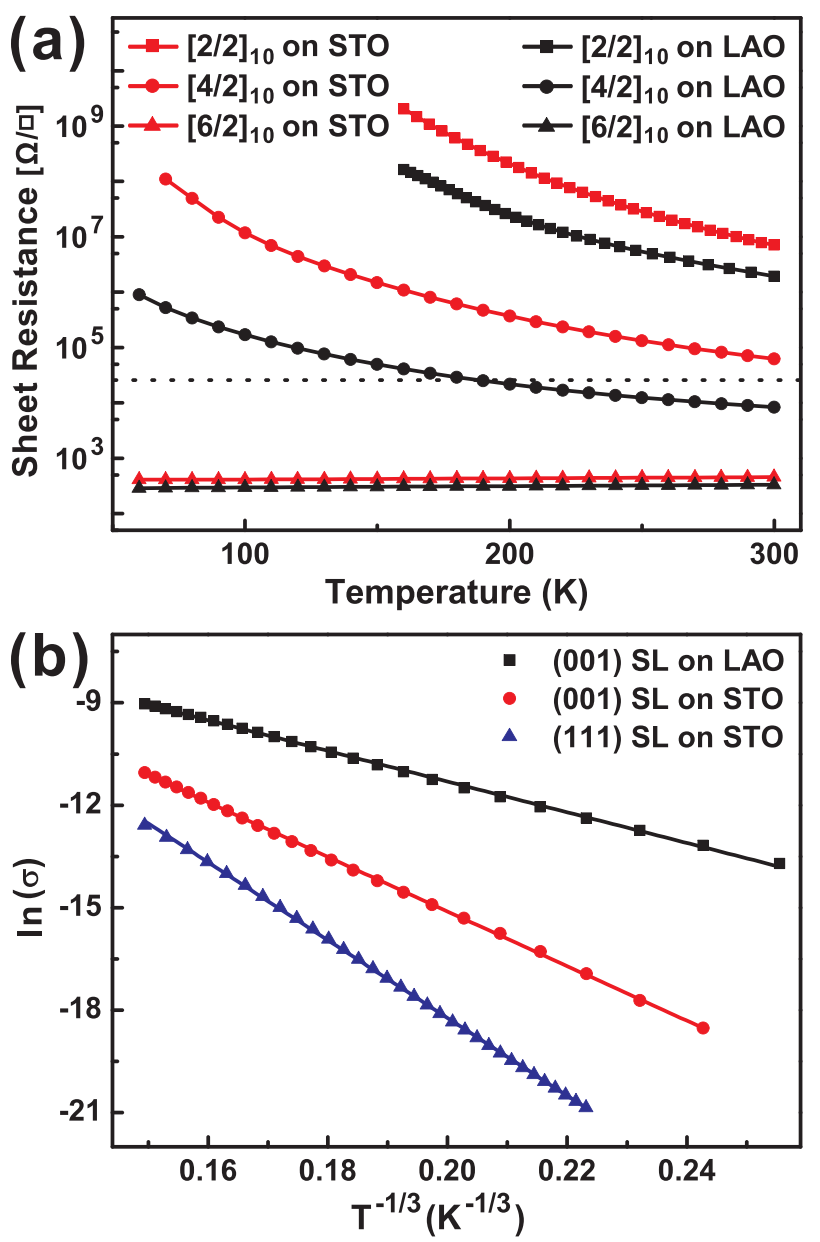

FIG. 4. (a) Temperature dependence of the sheet resistance of (001)-oriented SLs deposited on STO (red) and LAO (black) substrates. The horizontal black dotted line corresponds to the quantum of resistance in $2 \mathrm{D}$ state. (b) Logarithm of sheet conductance $\sigma$ (in units of $\mathrm{S} \square$ ) as a function of $1 / \mathrm{T}^{1 / 3}$ of $[4 / 2]_{10}$ SLs on LAO (001), STO (001) and STO (111) substrates. The lines are linear fitting, indicating 2D VRH-type conductivity. 
TABLE II. Fitting parameters and calculated values of the 2D VRH model for [4/2 $]_{10}$ SLs: substrate, sheet resistance $R_{S}$ at $300 \mathrm{~K}$, fit parameter $T_{0}$, the ratio of $R_{h o p} / a$ at $300 \mathrm{~K}$, hopping energy $E_{\text {hop }}$ with $T$ in $\mathrm{K}$, density of states at the Fermi lever $N\left(\mathrm{E}_{\mathrm{F}}\right)$, and localization length $a$.

\begin{tabular}{lccccc}
\hline \hline Substrate & $R_{S}(\mathrm{~T}=300 \mathrm{~K})\left(10^{5} \Omega / \square\right)$ & $T_{0}\left(10^{5} \mathrm{~K}\right)$ & $R_{\text {hop }} / a(\mathrm{~T}=300 \mathrm{~K})$ & $E_{\text {hop }}(\mathrm{meV})$ & $N\left(E_{\mathrm{F}}\right)\left(\mathrm{eV}^{-1} \mathrm{~cm}^{-2}\right)$ \\
\hline STO (001) & 3.70 & 5.10 & 3.98 & $2.30 T^{2 / 3}$ & $1.79 \times 10^{18}$ \\
LAO (001) & 0.836 & 9.07 & 4.82 & $1.29 T^{2 / 3}$ & 0.42 \\
STO (111) & 82.2 & 14.8 & 5.67 & $3.27 T^{2 / 3}$ & $1.74 \times 10^{16}$ \\
\hline \hline
\end{tabular}

In order to clarify the intrinsic conductivity mechanism of the electron-localized LNO/LMO SLs, the temperaturedependent conductance is fitted as a function of $T^{-1 / 3}$, as shown in Fig. 4(b). This behavior can be described with a 2D Mott variable range hopping (VRH) model $\sigma=\sigma_{0} \cdot \exp$ $\left[-\left(T_{0} / T\right)^{-1 / 3}\right]$, where $T_{0}$ is the localization temperature depending on the density of states $N\left(E_{F}\right)$ near the Fermi level. ${ }^{29}$ This model has been repeatedly confirmed being suitable for the description of electronic transport of LNObased SLs. ${ }^{13,22}$ Assuming that the Fermi energy lies in the range of the localized state and $N\left(E_{F}\right)$ values are on the same order of magnitude within several $k_{B} T$, we can make a rough approximation of $N\left(E_{\mathrm{F}}\right)$ using $k_{B} N\left(E_{F}\right)=\partial n / \partial T \approx n$ $(100 \mathrm{~K}) / 100 \mathrm{~K}^{30}$ Thus, the localization length $a$ can be obtained by inserting $T_{0}$ and $N\left(E_{F}\right)$ values into $a^{2}=13.8 /$ $k_{B} N\left(E_{F}\right) T_{0} \cdot{ }^{31}$ The calculated values of localization length $a$ of our SLs are reasonable in comparison to the in-plane $\mathrm{Ni}-\mathrm{O}$ bond distance in SLs. In addition, the hopping distance $R_{\text {hop }}$ and hopping energy $E_{\text {hop }}$ are given by $R_{h o p}=\frac{1}{3} a\left(\frac{T_{0}}{T}\right)^{1 / 3}$ and $E_{\text {hop }}=\frac{1}{3} k_{B} T^{2 / 3} T_{0}^{1 / 3}$, respectively. ${ }^{32,33}$ The fulfillment of the two requirements $R_{h o p} / a>1$ and $E_{h o p} / k_{B} T>1$ within the measured temperature range verifies the validity of the $2 \mathrm{D}$ VRH mechanism. All fitting parameters and calculated values listed in Table II are in a reasonable range. ${ }^{22}$ Our results provide the necessary quantitative experimental feedback to the theory for the above mentioned conductivity mechanism for LNO-based SLs. ${ }^{22,25}$

In summary, the in-plane lattice matched (111)- and (001)-oriented SLs consisting of paramagnetic LNO and ferromagnetic LMO were grown by PLD. The experimental evidence of exchange bias was found in SLs with both (001) and (111) orientations. This can be explained by charge transfer from $\mathrm{Mn}$ to $\mathrm{Ni}$ atoms, inducing an antiferromagnetic behavior. In addition, a metal-insulator transition was observed for decreasing LNO thickness. Strong localization appears when the LNO thickness in the superlattice is reduced to 4 unit cells, and the conductivity is dominated by $2 \mathrm{D}$ variable range hopping. The results are significant for a better understanding of the derivation of magnetic and insulating states in LNO SLs. The charge transfer-induced redistributed interface of $\mathrm{LNO} /$ LMO provides an intriguing platform for studying fundamental electronic and magnetic interaction in oxide heterostructures.

See supplementary material for additional Figures S1-S4 on surface morphology, out-of-plane and in-plane crystalline structure, and temperature dependent magnetic data, and temperature dependent sheet resistance of the (111)-oriented superlattices, respectively, as mentioned in the text.

This work is funded by the Deutsche Forschungsgemeinschaft (DFG) in the framework of
Collaborative Research Center SFB 762 "Functionality of Oxide Interfaces."

${ }^{1}$ D. van Delft and P. Kes, Phys. Today 63(9), 38-42 (2010).

${ }^{2}$ H. Y. Hwang, Y. Iwasa, M. Kawasaki, B. Keimer, N. Nagaosa, and Y. Tokura, Nature Mater. 11, 103-113 (2012).

${ }^{3}$ D. Doennig, S. Baidya, W. E. Pickeet, and R. Pentcheva, Phys. Rev. B 93, 165145 (2016).

${ }^{4}$ M. Kiwi, J. Magn. Magn. Mater. 234, 584-595 (2001).

${ }^{5}$ J. Nogués and I. K. Schuller, J. Magn. Magn. Mater. 192, 203-232 (1999).

${ }^{6}$ M. Gibert, P. Zubko, R. Scherwitzl, J. Íniguez, and J.-M. Triscone, Nature Mater. 11, 195-198 (2012).

${ }^{7}$ S. Dong and E. Dagotto, Phys. Rev. B 87, 195116 (2013).

${ }^{8}$ C. Piamonteze, M. Gibert, J. Heidler, J. Dreiser, S. Rusponi, H. Brune, J.M. Triscone, F. Nolting, and U. Staub, Phys. Rev. B 92, 014426 (2015).

${ }^{9}$ A. T. Lee and M. J. Han, Phys. Rev. B 88, 035126 (2013).

${ }^{10}$ S. Middey, P. Rivero, D. Meyers, M. Kareev, X. Liu, Y. Cao, J. W. Freeland, S. Barraza-Lopez, and J. Chakhalian, Sci. Rep. 4, 6819 (2014).

${ }^{11}$ S. Middey, D. Meyers, M. Kareev, E. J. Moon, B. A. Gray, X. Liu, J. W. Freeland, and J. Chakhalian, Appl. Phys. Lett. 101, 261602 (2012).

${ }^{12}$ R. L. Stamps, J. Phys. D: Appl. Phys. 33, R247-R268 (2000).

${ }^{13}$ H. M. Wei, M. Jenderka, M. Bonholzer, M. Grundmann, and M. Lorenz, Appl. Phys. Lett. 106, 042103 (2015).

${ }^{14}$ H. M. Wei, M. Grundmann, and M. Lorenz, Appl. Phys. Lett. 109, 082108 (2016).

${ }^{15}$ V. Lazenka, M. Lorenz, H. Modarresi, M. Bisht, R. Rüffer, M. Bonholzer, M. Grundmann, M. J. Van Bael, A. Vantomme, and K. Temst, Appl. Phys. Lett. 106, 082904 (2015).

${ }^{16}$ Z. Li, C. Jing, J. P. Chen, S. J. Yuan, S. X. Cao, and J. C. Zhang, Appl. Phys. Lett. 91, 112505 (2007).

${ }^{17}$ J. C. Rojas Sánchez, B. Nelson-Cheeseman, M. Granada, E. Arenholz, and L. B. Steren, Phys. Rev. B 85, 094427 (2012).

${ }^{18}$ K. Maaz, A. Mumtaz, S. K. Hasanain, and M. F. Bertina, J. Magn. Magn. Mater. 322, 2199-2202 (2010).

${ }^{19}$ Y. F. Chen, D. Spoddig, and M. Ziese, J. Phys. D: Appl. Phys. 41, 205004 (2008).

${ }^{20}$ E. F. Kneller and F. E. Luborsky, J. Appl. Phys. 34, 656 (1963).

${ }^{21}$ J. Barzola-Quiquia, A. Lessig, A. Ballestar, C. Zandalazini, G. Bridoux, F. Bern, and P. Esquinazi, J. Phys.: Condens. Matter 24, 366006 (2012).

${ }^{22}$ J. Hoffman, I. C. Tung, B. B. Nelson-Cheeseman, M. Liu, J. W. Freeland, and A. Bhattacharya, Phys. Rev. B 88, 144411 (2013).

${ }^{23}$ G. P. Mambrini and E. R. Leite, J. Appl. Phys. 102, 043708 (2007).

${ }^{24}$ R. Scherwitzl, P. Zubko, C. Lichtensteiger, and J.-M. Triscone, Appl. Phys. Lett. 95, 222114 (2009).

${ }^{25}$ A. V. Boris, Y. Matiks, E. Benckiser, A. Frano, P. Popovich, V. Hinkov, P. Wochner, M. C. Colin, E. Detemple, V. K. Malik, C. Bernhard, T. Prokscha, A. Suter, Z. Salman, E. Morenzoni, G. Cristiani, H. U. Habermeier, and B. Keimer, Science 332, 937-940 (2011).

${ }^{26}$ D. C. Licciardello and D. J. Thouless, Phys. Rev. Lett. 35, 1475 (1975).

${ }^{27}$ R. Scherwitzl, S. Gariglio, M. Gabay, P. Zubko, M. Gibert, and J.-M. Triscone, Phys. Rev. Lett. 106, 246403 (2011).

${ }^{28}$ M. Kawai, S. Inoue, M. Mizumaki, N. Kawamura, N. Ichikawa, and Y. Shimakawa, Appl. Phys. Lett. 94, 082102 (2009).

${ }^{29}$ W. Brenig, Philos. Mag. 27, 1093 (1973).

${ }^{30}$ G. Eda, C. Mattevi, H. Yamaguchi, H. Kim, and M. Chhowalla, J. Phys. Chem. C 113, 15768 (2009).

${ }^{31}$ M. I. B. Utama, X. Lu, D. Zhan, S. T. Ha, Y. Yuan, Z. Shen, and Q. Xiong, Nanoscale 6, 12376-12382 (2014).

${ }^{32}$ Z. H. Khan, M. Husain, T. P. Perng, N. Salah, and S. Habib, J. Phys.: Condens. Matter 20, 475207 (2008).

${ }^{33}$ S. J. Lee and J. B. Ketterson, Phys. Rev. B 46, 12695 (1992). 